
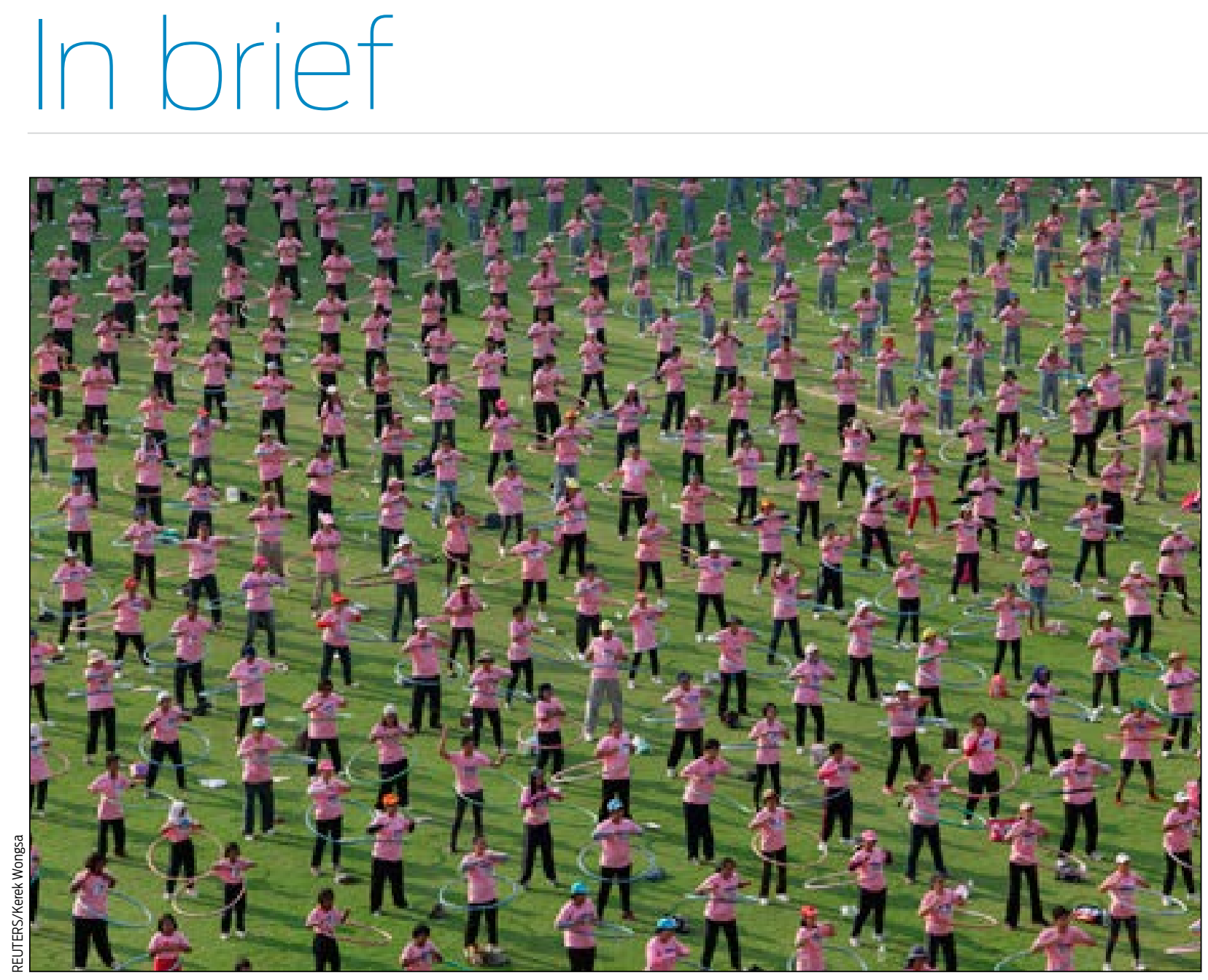

It started out as a public health program to promote exercise and good health, and ended up as an attempt to break the Guinness world record for the most people simultaneously hula-hooping. Thailand broke the record previously held by Taiwan with a new record of 4483 people hula-hooping for 7 minutes. The event was held at the Thammasat University stadium in Pathum Thani province, on the outskirts of Bangkok.

\title{
From the CSIRO Preventative Health Flagship
}

\section{Chasing the "ghost in our genes" to beat obesity}

Australian scientists are investigating how epigenetics influences the development of obesity and related diseases, such as diabetes. The 4-year EpiSCOPE project involves the CSIRO (Commonwealth Scientific and Industrial Research Organisation), the Garvan Institute of Medical Research, the Women's and Children's Health Research Institute, and the University of South Australia, and is supported by the Science and Industry Endowment Fund.

The EpiSCOPE team is investigating the function of a set of molecular signals that determine the way in which information in the genome is used. Collectively known as the epigenome, they emerge in utero and during early infancy and control normal development. These control signals are influenced by the individual's environment and exposures before birth and in early life - potentially affecting both the individual and his or her descendants decades later. Described as the "ghost in our genes", the epigenome is effectively a "molecular memory" or signature of these exposures. Accumulating evidence suggests that these molecules influenced by nutritional and environmental exposures affect our risk of obesity and other diseases of metabolism later in life.

The team is aiming to build the first map of a normal fat cell's epigenome, to identify the signatures associated with the development of
Peter Molloy

Research Stream Leader

Katherine Samaras

Senior Research Fellow,

Garvan Institute of

Medical Research

Beverly Muhlhausler

Senior Research Fellow,

Women's and Children's

Health Research Institute

Caroline McMillen

Professor, University of

South Australia

CSIRO Preventative Health Flagship

doi: 10.5694/mjal3.10098 obesity in children and adults, and to characterise how maternal obesity and poor nutrition, as well as specific nutrients, may shape the developing epigenome. A key goal is to identify biomarkers and develop diagnostic tests to predict an individual's risk of obesity, diabetes and other metabolic disorders, to assist interventions for maintaining a healthy weight.

The detection of predictive biomarkers provides the opportunity for intervention long before obesity becomes established, rather than after its onset, when many interventions fail. Such new strategies are urgently needed if we are to curtail the emerging obesity epidemic, with its current cost to the Australian community of $\$ 8$ billion per year. 


\section{Survival time from lung cancer in six countries}

Lung cancer survival varied widely across six wealthy nations, including Australia, from 2004 to 2007, according to a new study published in Thorax. Population-based data for adults (aged 15-99 years) diagnosed with lung cancer in regional and national cancer registries in Australia, Canada, Denmark, Norway, Sweden and the UK were collected, to consider whether differences in stage at diagnosis could fully explain the variations in survival rates between countries. Age-standardised 1-year net survival from non-small cell lung cancer ranged from $30 \%$ in the UK to $46 \%$ in Sweden. Australia's rate was $42 \%$. The authors concluded that, while differences in stage at diagnosis explained some of the variation in survival rates, "wide disparities in stage-specific survival exist, suggesting that other factors are also important such as differences in treatment". The authors wrote that "stage should be included in international cancer studies, and the comparability of population-based data should be improved".

\section{Thorax 2013; 11 February (online)}

doi: 10.1136/thoraxjnl-2012-202297

\section{Chinese pig manure linked to antibiotic resistance}

Chinese pig manure, unmonitored and unregulated for antibiotic and metal content, is a public health hazard and major source of antibiotic resistance genes (ARGs), according to a new study in PNAS Early Edition. China is the world's largest antibiotics producer and consumer, producing 210 million kilograms in 2007 alone, $41 \%$ of which was used in livestock industries. The authors wrote that "manure is a major source of antibiotic pollution ... and China produces an estimated 618 billion kilograms of swine manure annually". Samples were collected from farms in three Chinese provinces, then DNA was extracted and genetic analysis (using high-capacity quantitative polymerase chain reaction arrays) undertaken. The authors found 149 unique ARGs, and many ARGs often coexisted in one manure sample, potentially increasing the risk of co-resistance to multiple antibiotics and their transfer between multiple bacterial species. The researchers also found elevated levels of zinc, copper, arsenic and antibiotics which correlated with ARG levels in the samples. "The diverse set of resistance genes detected potentially confer resistance to all major classes of antibiotics, including antibiotics critically important for human medicine", they wrote, describing the results as "alarming". They warned of the possible expansion of "a local contamination to regional and even global scales".

PNAS Early Edition 2013; 11 February (online)

doi: 10.1073/pnas.1222743110

\section{From the MJA archives}

\section{MJA 1958; 11 January (edited extract)}

\section{Death of Mozart}

In the Wiener Medizinische Wochenschrift of 22

December 1956, there appeared an article by D. Kerner reviving an old rumour that Mozart had been poisoned by the composer and theatre director, Antonio Salieri, and diagnosing the toxic agent as mercury. [Six months later, German dermatologist and music historian] Aloys Greither is merciless in his exposure of the faulty scholarship of the Vienna writer. The relations between Mozart and Salieri are now shown to be not only correct but friendly, and furthermore there is mighty little in the condition of the dying musician which suggests mercury poisoning, no salivation nor any loosening of teeth. The fatal condition was clearly uraemia of renal origin. Greither administers a severe and, many will think, well merited rebuke to Kerner for not taking ordinary precautions to verify his alleged charges. This dismissal of the poison story and exoneration of Salieri will be accepted as definitive and final.

Editorial

\section{High-risk NSAIDs remain popular worldwide}

Diclofenac should be removed from essential medicine lists (EMLs) worldwide, according to the authors of a report published in PLOS Medicine. Using data from published meta-analyses, the authors correlated the relative risk of cardiovascular events associated with specific non-steroidal anti-inflammatory drugs (NSAIDs) with the EMLs of 100 countries and sales information for NSAIDs in 15 countries. Apart from rofecoxib, which was removed from EMLs worldwide 8 years ago, diclofenac was the NSAID most associated with an increased risk of cardiovascular events $(40 \%-60 \%$ higher relative risk compared with non-use). Yet it remains on the EMLs of 74 countries, including Australia. In contrast, naproxen, rated the safest of the NSAIDs, features on only 27 EMLs. An accompanying editorial said that "emerging evidence about NSAID risk is poorly translated into practice and sales in countries around the world, raising questions about the use and promotion of potentially harmful drugs".

PLoS Med 2013; 10 (2): el001388 doi: 10.1371/journal.pmed.1001388 PLoS Med 2013; 10 (2): el001389 doi: 10.1371/journal.pmed.1001389

\section{H. pylori eradication factors}

Non-adherence and demographics may be as important as specific antibiotic regimens in the eradication of Helicobacter pylori in gastric cancer highincidence regions such as Latin America, a new study published in JAMA has found. The authors conducted a cohort analysis of 1463 participants aged 21 to 65 years from seven Latin American communities, who were randomly allocated to one of three treatment groups - triple therapy, sequential and concomitant — and observed between September 2009 and July 2011. Participants with a positive C-urea breath test 6 to 8 weeks after treatment were offered retreatment. All participants were followed up 1 year after treatment. The estimated 1-year eradication success rate was $80.4 \%$ (triple), $79.8 \%$ (sequential) and $77.8 \%$ (concomitant). However, significant predictors of successful eradication of $H$. pylori after 1 year were the study site, male sex, older age and adherence to initial therapy. Younger women with more than three children in their household at the Colombian site had the lowest success rates.

JAMA 2013; 309: 578-586 doi: 10.1001/jama.2013.311 